Homology, Homotopy and Applications, vol. 7(1), 2005, pp.185-195

\title{
WHAT DOES THE CLASSIFYING SPACE OF A CATEGORY CLASSIFY?
}

\author{
MICHAEL WEISS \\ (communicated by Graham Ellis)
}

\begin{abstract}
The classifying space of a small category classifies sheaves whose values are contravariant functors from that category to sets and whose stalks are representable.
\end{abstract}

\section{Introduction}

Let $\mathcal{C}$ be a small category. Contravariant functors from $\mathcal{C}$ to the category of sets, and natural transformations between them, will be called $\mathcal{C}$-sets and $\mathcal{C}$-maps, respectively. The category of $\mathcal{C}$-sets shares many good properties with the category of sets. (In short, it is a topos. See [4] or [7]. Here we will not make any explicit use of this fact.) The $\mathcal{C}$-sets which are of the form $b \mapsto \operatorname{mor}_{\mathcal{C}}(b, c)$ for fixed $c \in \mathcal{C}$, and any isomorphic ones, are called representable. By the Yoneda lemma, the representable $\mathcal{C}$-sets form a full subcategory of the category of all $\mathcal{C}$-sets which is equivalent to $\mathcal{C}$.

We will be concerned with sheaves of $\mathcal{C}$-sets on a topological space $X$. For such a sheaf, and $x \in X$, the stalk $\mathscr{F}_{x}$ is again a $\mathcal{C}$-set. It is the direct limit of the $\mathcal{C}$-sets $\mathscr{F}(U)$ where $U$ runs through the open neighborhoods of $x$.

Theorem 0.1. The classifying space $B \mathcal{C}$ classifies sheaves of $\mathcal{C}$-sets with representable stalks.

\section{Notation, terminology, clarifications.}

Let $\mathscr{F}$ be any sheaf of $\mathcal{C}$-sets on $X$. We may regard $\mathscr{F}$ as a contravariant functor $(c, U) \mapsto \mathscr{F}^{(c)}(U)$ in two variables (where $c \in \mathrm{ob}(\mathcal{C})$ and $U$ is open in $\left.X\right)$. Specializing one of the variables, we obtain $\mathscr{F}^{(c)}$, a sheaf of sets on $X$, and $\mathscr{F}(U)$, a $\mathcal{C}$-set.

Let $\mathscr{L}$ be any sheaf of sets on $X$. The espace étalé of $\mathscr{L}$, denoted $\operatorname{Spé}(\mathscr{L})$, is the (disjoint) union of the stalks $\mathscr{L}_{x}$, suitably topologized. See [2, II.1, ex.1.13] for details. The sheaf $\mathscr{L}$ can be identified with the sheaf of continuous (partial) sections of the projection $\operatorname{Spé}(\mathscr{L}) \rightarrow X$.

The projection Spé $(\mathscr{L}) \rightarrow X$ is an étale map alias local homeomorphism. [But it happens frequently that $X$ is Hausdorff while Spé $(\mathscr{L})$ is not.] The construction Spé $(\mathscr{L})$ leads to a good notion of pullback of sheaves: for a map $v: Y \rightarrow X$, the

Partially supported by the Royal Society.

Received February 4, 2005, revised June 18, 2005; published on November 1, 2005.

2000 Mathematics Subject Classification: 57T30, 18F20, 18G55

Key words and phrases: category, classifying space, sheaf, representable functor

(C) 2005, Michael Weiss. Permission to copy for private use granted. 
pullback $v^{*} \mathscr{L}$ is defined in such a way that $\operatorname{Spé}\left(v^{*} \mathscr{L}\right)=v^{*} \operatorname{Spé}(\mathscr{L})$. More generally, for a sheaf $\mathscr{F}$ of $\mathcal{C}$-sets on $X$ and $v: Y \rightarrow X$, the pullback $v^{*} \mathscr{F}$ is defined in such a way that $\operatorname{Spé}\left(\left(v^{*} \mathscr{F}\right)^{(c)}\right)=v^{*} \operatorname{Spé}(\mathscr{F}(c))$.

Let $\mathscr{F}$ and $\mathscr{G}$ be sheaves of $\mathcal{C}$-sets on $X$, both with representable stalks. Let $e_{0}, e_{1}: X \rightarrow X \times[0,1]$ be given by $e_{0}(x)=(x, 0)$ and $e_{1}(x)=(x, 1)$. The sheaves $\mathscr{F}$ and $\mathscr{G}$ are concordant if there exists a sheaf of $\mathcal{C}$-sets $\mathscr{H}$ on $X \times[0,1]$, again with representable stalks, such that $e_{0}^{*} \mathscr{H} \cong \mathscr{F}$ and $e_{1}^{*} \mathscr{H} \cong \mathscr{G}$.

The precise meaning of theorem 0.1 is as follows. Suppose that $X$ has the homotopy type of a $\mathrm{CW}$-space. There is a natural bijection from the homotopy set $[X, B \mathcal{C}]$ to the set of concordance classes of sheaves of $\mathcal{C}$-sets on $X$ with representable stalks.

Remark. Suppose that $\mathcal{C}$ is a group. To be more precise, suppose that $\mathcal{C}$ has just one object $c$ and $\operatorname{mor}(c, c)$ is a group. Let $\mathscr{F}$ be a sheaf of $\mathcal{C}$-sets on a space $X$. If the stalks of $\mathscr{F}$ are all representable, then the projection $\operatorname{Spé}(\mathscr{F}) \rightarrow X$ is a principal mor $(c, c)$-bundle. Indeed any choice of an open $U$ and $s \in \mathscr{F}^{(c)}(U)$ determines a bundle chart

$$
\operatorname{Spé}(\mathscr{F} \mid U) \cong \operatorname{mor}(c, c) \times U .
$$

In this situation, concordant sheaves of $\mathcal{C}$-sets on $X$ (with representable stalks) are isomorphic, because "concordant" implies "isomorphic" for principal mor $(c, c)$ bundles.

Remark. The question in the title undoubtedly has many correct answers and a few have already been given elsewhere. Moerdijk [7, Introd.] has a result like theorem 0.1 in which the representability condition on stalks is replaced by a weaker condition, that of being principal. To explain what a principal $\mathcal{C}$-set is, we start with the following standard definitions:

- The transport category of a $\mathcal{C}$-set $S$ has objects $(c, x)$ where $c$ is an object of $\mathcal{C}$ and $x \in S(c)$. A morphism from $(c, x)$ to $(d, y)$ is a morphism $g: c \rightarrow d$ in $\mathcal{C}$ such that the induced map $S(g): S(d) \rightarrow S(c)$ takes $y$ to $x$. (Some people would call this the opposite of the transport category of $S$.)

- A category $\mathcal{D}$ is filtered if

- it has at least one object;

- for any two objects $d_{1}, d_{2}$ in $\mathcal{D}$ there exists another object $d_{3}$ and morphisms $d_{1} \rightarrow d_{3}, d_{2} \rightarrow d_{3}$;

- for any two morphisms in $\mathcal{D}$ with the same source and target, say $f, g: a \rightarrow$ $b$, there exists a coequalizer (a morphism $h: b \rightarrow c$ in $\mathcal{D}$ such that $h f=h g$ ).

Now a $\mathcal{C}$-set is principal if its transport category is filtered. (This is not exactly the terminology which Moerdijk uses. He calls a sheaf of $\mathcal{C}$-sets on $X$ a principal $\mathcal{C}^{\mathrm{op}}$-bundle if the transport category of each stalk, as defined above, is filtered.) A representable $\mathcal{C}$-set $S$ is certainly principal, since the transport category of $S$ has a terminal object. The converse does not hold.

For example, suppose that $\mathcal{C}$ itself is a filtered category which does not have a terminal object. Define a $\mathcal{C}$-set $S$ in such a way that $S(c)$ has exactly one element, for every object $c$ in $\mathcal{C}$. Then the transport category of $S$ is equivalent to $\mathcal{C}$, so $S$ is 
principal. But $S$ is not representable, since a representing object would be a terminal object for $\mathcal{C}$. It follows that there exist sheaves of $\mathcal{C}$-sets on some spaces $X$ which do not satisfy the condition of theorem 0.1 but which are principal $\mathcal{C}^{\text {op }}$-bundles according to Moerdijk's definition. (Take $X$ to be a point.)

Moerdijk [7] takes the discussion much further by considering topological categories, which I have not attempted to do.

Another precursor of theorem 0.1 is due to tom Dieck (1972, unpublished). He used a notion of $\mathcal{C}$-bundle defined in terms of a bundle atlas. His result was rediscovered in [5, thm 4.1.2].

Theorem 0.1 is anticipated and illustrated to some extent in [6].

\section{The canonical sheaf on $B C$}

We are going to construct a sheaf $\mathscr{E}$ of $\mathcal{C}$-sets on $B \mathcal{C}$ which will eventually turn out to be "universal". Recall to begin with that $B \mathcal{C}$ is the geometric realization of the simplicial set whose $n$-simplices are the contravariant functors $[n] \rightarrow \mathcal{C}$, where $[n]$ is the linearly ordered set $\{0,1,2, \ldots, n\}$. (There are historical reasons for insisting on contravariant functors $[n] \rightarrow \mathcal{C}$; the formulae for boundary operators look more familiar in the case where $\mathcal{C}$ is a group or monoid.) Now suppose that $U$ is open in $B \mathcal{C}$ and $c$ is an object of $\mathcal{C}$.

Definition 1.1. An element of $\mathscr{E}(c)(U)$ is a "function" which to every $\alpha:[n]^{\mathrm{op}} \rightarrow \mathcal{C}$ and $x \in B[n]^{\mathrm{op}} \cong \Delta^{n}$ with $\alpha_{*} x \in U$ assigns a morphism $s(\alpha, x): c \rightarrow \alpha(0)$ in $\mathcal{C}$. The function is required to be

- locally constant in the second variable, so that for $y \in \Delta^{n}$ sufficiently close to $x$, with $\alpha_{*} y \in U$, we have $s(\alpha, y)=s(\alpha, x)$;

- natural in the first variable. That is, for an order-preserving $g:[m] \rightarrow[n]$ and $y \in \Delta^{m}$, we have

$$
s\left(\alpha, g_{*} y\right)=\alpha(0, g(0)) \circ s(\alpha g, y)
$$

where $\alpha(0, g(0)): \alpha(g(0)) \rightarrow \alpha(0)$ is the morphism in $\mathcal{C}$ induced by the unique morphism $0 \rightarrow g(0)$ in $[n]$.

The contravariant dependence of $\mathscr{E}(c)(U)$ on $U$ and $c$ is obvious. The sheaf property is also obvious. Because of the naturality condition, an element $s$ of $\mathscr{E}^{(c)}(U)$ is determined by its values $s(\alpha, x)$ for nondegenerate $\alpha:[n]^{\text {op }} \rightarrow \mathcal{C}$ and $x \in \Delta^{n} \backslash \partial \Delta^{n}$. Then $\alpha$ and $x$ are determined by $\alpha_{*} x \in U$; in particular $n$ is the dimension of the cell (in the canonical CW-decomposition of $B \mathcal{C}$ ) to which $\alpha_{*} x$ belongs. (Regarding cells, the convention used here is that the cells of a CW-space are pairwise disjoint, and each cell is homeomorphic to some euclidean space. This is in agreement with [1], for example.)

Lemma 1.2. Fix a nondegenerate $\beta:[m]^{\mathrm{op}} \rightarrow \mathcal{C}$ and $y \in \Delta^{m} \backslash \partial \Delta^{m}$. The stalk of $\mathscr{E}$ at $\beta_{*} y$ is the contravariant functor $c \mapsto \operatorname{mor}(c, \beta(0))$.

Proof. Any point of $B \mathcal{C}$ can be uniquely written as $\alpha_{*} z$ where $\alpha:[n]^{\text {op }} \rightarrow \mathcal{C}$ is nondegenerate and $z \in \Delta^{n} \backslash \partial \Delta^{n}$. If $\alpha_{*} z$ is sufficiently close to $\beta_{*} y$, then some 
degeneracy of $\beta$ will be a face of $\alpha$. That is, there are an order-preserving surjection $f:[k] \rightarrow[m]$ and an order-preserving injection $g:[k] \rightarrow[n]$ such that $\alpha g=\beta f$. And moreover, there will be $w \in \Delta^{k}$ such that $f_{*} w=y$ and $z$ is close to $g_{*} w$. For $s$ in the stalk of $\mathscr{E}^{(c)}$ at $\beta_{*} y \in B \mathcal{C}$, we then have

$$
\begin{aligned}
& s(\alpha, z)=s\left(\alpha, g_{*} w\right)=\alpha(0, g(0)) \circ s(\alpha g, w), \\
& s(\alpha g, w)=s(\beta f, w)=s\left(\beta, f_{*} w\right)=s(\beta, y),
\end{aligned}
$$

so that $s(\alpha, z)=\alpha(0, g(0)) \circ s(\beta, y)$. Hence $s$ is determined by $s(\beta, y) \in \operatorname{mor}(c, \beta(0))$. To establish the existence of a germ $s$ with prescribed value $s(\beta, y)$, we proceed differently. Suppose inductively that the values of $s$ at points near $\beta_{*} y$ and in the $(n-1)$-skeleton of $B C$ have already been determined consistently, for some fixed $n>m$. For an $n$-simplex $\alpha:[n]^{\mathrm{op}} \rightarrow \mathcal{C}$ we have an attaching map from $\partial \Delta^{n}$ to the $(n-1)$-skeleton of $B \mathcal{C}$. Hence $s(\alpha, x)$ is already determined for $x$ in some open subset $V$ of $\partial \Delta^{n}$. As a function on $V$, denoted informally $s \mid V$, it satisfies the continuity and naturality conditions of definition 1.1 (mutatis mutandis). We now have to find an open $W \subset \Delta^{n}$ such that $V=W \cap \partial \Delta^{n}$ and an extension of $s \mid V$ from $V$ to $W$. This is easy. For example, $\Delta^{n}$ can be identified with a cone on $\partial \Delta^{n}$ and $W$ could then be defined as the cone on $V$ minus the cone point. Then $s \mid V$ has a unique extension from $V$ to $W$.

For an object $c$ of $\mathcal{C}$, let $(c \downarrow \mathcal{C})$ be the "under" category associated with $c$. The objects of $(c \downarrow \mathcal{C})$ are the morphisms in $\mathcal{C}$ with source $c$, and the morphisms of $(c \downarrow \mathcal{C})$ are morphisms in $\mathcal{C}$ under $c$. The classifying space $B(c \downarrow \mathcal{C})$ is contractible since $(c \downarrow \mathcal{C})$ has an initial object. The forgetful map $B(c \downarrow \mathcal{C}) \rightarrow B \mathcal{C}$ has a canonical factorization

$$
B(c \downarrow \mathcal{C}) \stackrel{\lambda_{c}}{\longrightarrow} \operatorname{Spé}(\mathscr{E}(c)) \stackrel{\text { proj. }}{\longrightarrow} B \mathcal{C} .
$$

Indeed, any point of $B \mathcal{C}$ can be uniquely written as $\alpha_{*} z$ where $\alpha:[n]^{\text {op }} \rightarrow \mathcal{C}$ is nondegenerate and $z \in \Delta^{n} \backslash \partial \Delta^{n}$. Lifting $\alpha_{*} z$ to $B(c \downarrow C)$ amounts to specifying a morphism $c \rightarrow \alpha(n)$ in $\mathcal{C}$; lifting $\alpha_{*} z$ to Spé $(\mathscr{E}(c))$ amounts to specifying a morphism $c \rightarrow \alpha(0)$ in $\mathcal{C}$. Clearly a morphism $c \rightarrow \alpha(n)$ determines a morphism $c \rightarrow \alpha(0)$ by composition with $\alpha(0, n): \alpha(n) \rightarrow \alpha(0)$.

The map $\lambda_{c}$ will be useful in the proof of

Proposition 1.3. The space Spé( $\left.\mathscr{E}^{(c)}\right)$ is weakly contractible.

Proof. Let $\overline{B C}$ be the fat realization of the nerve of $\mathcal{C}$, obtained by ignoring the degeneracy operators. The quotient map $q: \overline{B C} \rightarrow B \mathcal{C}$ is a quasifibration with contractible fibers. To see this, note that the fat realization of any simplicial set $Z$ can be described as the ordinary realization of another simplicial set $\bar{Z}$ whose $n$ simplices are triples $(k, f, x)$ where $x \in Z_{k}$ and $f:[n] \rightarrow[k]$ is an order-preserving surjection. The forgetful simplicial map $\bar{Z} \rightarrow Z$ is a Kan fibration with contractible fibers; hence the induced map of (lean) geometric realizations, $|\bar{Z}| \rightarrow|Z|$, is a quasifibration with contractible fibers. See $[\mathbf{3}]$.

Let $E=\operatorname{Spé}\left(\mathscr{E}^{(c)}\right)$, let $r: E \rightarrow B \mathcal{C}$ be the projection, and let $\bar{E}=q^{*} E$. In the 
pullback square

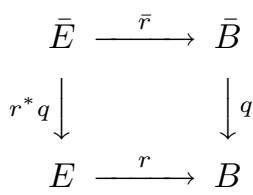

the map $q$ is a quasifibration with contractible fibers and $r$ is a local homeomorphism. It follows that $r^{*} q$ is a quasifibration with contractible fibers, and consequently a weak homotopy equivalence.

It remains to prove that $\bar{E}$ is contractible, or equivalently, that the canonical map $\bar{\lambda}_{c}: \bar{B}(c \downarrow C) \rightarrow \bar{E}$, the fat version of $\lambda_{c}$, is a weak homotopy equivalence. Suppose therefore that $Y$ is any finitely generated $\Delta$-set (= "simplicial set without degeneracy operators") and let

$$
f:|Y| \rightarrow \bar{E}
$$

be any map. We want to show that, up to a homotopy, $f$ lifts to $\bar{B}(c \downarrow C)$. The argument has two parts.

(i) If $\bar{r} f:|Y| \rightarrow \bar{B}$ is induced by a map of the underlying $\Delta$-sets, then $f$ admits a unique factorization through $\bar{B}(c \downarrow C)$.

(ii) Modulo iterated barycentric subdivision of $|Y|$, and a homotopy of $f$, the composition $\bar{r} f$ is indeed induced by a map of the underlying $\Delta$-sets.

For the proof of (i), we may assume that $Y$ is generated by a single $n$-simplex, so $|Y|=\Delta^{n}$. Suppose that $\bar{r} f: \Delta^{n} \rightarrow B \mathcal{C}$ is the characteristic map of an $n$-simplex $\alpha:[n]^{\text {op }} \rightarrow \mathcal{C}$ in the nerve of $\mathcal{C}$. The extra information contained in $f$ amounts to compatible morphisms $u_{i}: c \rightarrow \alpha(i)$ for $i=0,1, \ldots, n$; clearly all $u_{i}$ are determined by $u_{n}$. Together, $u_{n}$ and $\alpha$ determine an $n$-simplex in the nerve of $(c \downarrow \mathcal{C})$.

For the proof of (ii), we note that the first barycentric subdivision of $\bar{B} \mathcal{C}$ can be described as $\bar{B} \mathcal{C}^{\prime}$ for another category $\mathcal{C}^{\prime}$. An object of $\mathcal{C}^{\prime}$ is a simplex of the nerve of $\mathcal{C}$; a morphism from an $m$-simplex $\alpha$ to an $n$-simplex $\beta$ is an injective order-preserving $v:[m] \rightarrow[n]$ with $v^{*} \beta=\alpha$. The functor $\mathcal{C}^{\prime} \rightarrow \mathcal{C}$ given by $\alpha \mapsto \alpha(0)$ induces a $\Delta$-map from the nerve of $\mathcal{C}$ to the nerve of $\mathcal{C}^{\prime}$, and then a map

$$
\varphi_{1}: \bar{B} \mathcal{C} \rightarrow \bar{B} \mathcal{C}^{\prime} .
$$

This map is not a homeomorphism. There is of course another (well-known) map $\varphi_{0}: \bar{B} \mathcal{C}^{\prime} \rightarrow B \mathcal{C}$ which is a homeomorphism. What is important here is that $\varphi_{0}$ and $\varphi_{1}$ are homotopic in an obvious way, by a homotopy $\left(\varphi_{t}\right)_{t \in[0,1]}$. (Each track of the homotopy is a straight line segment, or a single point, in a simplex of $\bar{B} \mathcal{C}$.) The homotopy $\left(\varphi_{t} \varphi_{0}^{-1}\right)_{t \in[0,1]}$, from the identity of $\bar{B} \mathcal{C}$ to $\varphi_{1} \varphi_{0}^{-1}$, has a unique lift to a homotopy

$$
\left(\psi_{t}: \bar{E} \times[0,1] \rightarrow \bar{E}\right)_{t \in[0,1]}
$$

with $\psi_{0}=$ id. (To verify this claim, compare the pullbacks of $\bar{E}$ under the maps

$$
\bar{B} \mathcal{C} \times[0,1] \stackrel{\varphi \varphi_{0}^{-1}}{\longrightarrow} \overline{B C}, \quad \bar{B} \mathcal{C} \times[0,1] \stackrel{\text { proj. }}{\longrightarrow} \bar{B} \mathcal{C} .
$$

They are homeomorphic as spaces over $\bar{B} \mathcal{C} \times[0,1]$.) We can similarly look at iterated 
barycentric subdivisions of $\overline{B C}$. They all have two canonical maps $\varphi_{0}, \varphi_{1} \overline{B C}$, one being a homeomorphism and the other being simplicial, and these two maps are homotopic by a homotopy $\left(\varphi_{t}\right)_{t \in[0,1]}$. Again, the homotopy $\left(\varphi_{t} \varphi_{0}^{-1}\right)_{t \in[0,1]}$ has a unique lift to a homotopy $\left(\psi_{t}: \bar{E} \times[0,1] \rightarrow \bar{E}\right)_{t \in[0,1]}$ with $\psi_{0}=$ id. Coming back now to maps $|Y| \rightarrow \bar{E}$, any such map is homotopic to a map $f$ such that $\bar{r} f$ is induced by a $\Delta$-map from some iterated barycentric subdivision of $Y$ to some iterated barycentric subdivision of the nerve of $\mathcal{C}$. Compose $f$ with $\psi_{1}$ from the above homotopy. Then $\bar{r} \psi_{1} f$ is induced by a $\Delta$-map from $Y$ to the nerve of $\mathcal{C}$.

\section{Resolutions}

The previous section gives us a method to "convert" a map $f: X \rightarrow B \mathcal{C}$ into a sheaf of $\mathcal{C}$-sets on $X$ with representable stalks, by $f \mapsto f^{*} \mathscr{E}_{\mathcal{C}}$. Going in the opposite direction is more difficult. From a sheaf $\mathscr{F}$ of $\mathcal{C}$-sets on $X$, we shall construct a "resolution" $p_{\mathscr{F}}: X_{\mathscr{F}} \rightarrow X$ and a map $\pi_{\mathscr{F}}: X_{\mathscr{F}} \rightarrow B \mathcal{C}$. It turns out that $p_{\mathscr{F}}$ is a homotopy equivalence if $\mathscr{F}$ has representable stalks and $X$ is a $\mathrm{CW}$-space. Then we can choose a homotopy inverse $p_{\mathscr{F}}^{-1}$ and obtain a map $\pi_{\mathscr{F}} p_{\mathscr{F}}^{-1}: X \rightarrow B \mathcal{C}$, well defined up to homotopy.

Let $\mathcal{O}(X)$ be the poset of open subsets of a space $X$, ordered by inclusion. Let $\mathscr{F}$ be a sheaf of $\mathcal{C}$-sets on $X$. We can regard $\mathscr{F}$ as a contravariant functor from $\mathcal{O}(X) \times \mathcal{C}$ to sets. The functor $\mathscr{F}$ determines a transport category $\mathcal{T}_{\mathscr{F}}$ whose objects are the triples $(U, c, s)$ consisting of an object $U$ in $\mathcal{O}(X)$, an object $c$ in $\mathcal{C}$, and $s \in \mathscr{F}^{(c)}(U)$. A morphism from $(U, c, s)$ to $(V, d, t)$ is a morphism $U \rightarrow V$ in $\mathcal{O}(X)$ together with a morphism $f: c \rightarrow d$ in $\mathcal{C}$ such that $f^{*}(t) \mid U=s$. Let $\tau$ be the tautological functor (taking $U \in \mathcal{O}(X)$ to the space $U$ ) from $\mathcal{O}(X)$ to spaces, and let $\varphi: \mathcal{T}_{\mathscr{F}} \rightarrow \mathcal{O}(X)$ be the forgetful functor. Put

$$
X_{\mathscr{F}}:=\operatorname{hocolim} \tau \varphi .
$$

This comes with a canonical projection $p_{\mathscr{F}}: X_{\mathscr{F}} \rightarrow X$, induced by the obvious natural inclusions $\tau \varphi(U, c, s) \rightarrow X$. There is also a projection $X_{\mathscr{F}} \rightarrow B \mathcal{T}_{\mathscr{F}}$ which we can compose with the forgetful map $B \mathcal{T}_{\mathscr{F}} \rightarrow B \mathcal{C}$. This gives

$$
\pi_{\mathscr{F}}: X_{\mathscr{F}} \rightarrow B \mathcal{C} .
$$

Proposition 2.1. If $\mathscr{F}$ has representable stalks, then the projection $p_{\mathscr{F}}: X_{\mathscr{F}} \rightarrow X$ is a weak homotopy equivalence.

The proof relies on a few lemmas which in turn rely on the notion of a microfibration. Recall that a map $p: E \rightarrow B$ is a Serre fibration if it has the homotopy lifting property for homotopies $X \times[0,1] \rightarrow B$, with prescribed "initial" lift $X \rightarrow E$, where $X$ is a CW-space. [It is enough to check this in all cases where $X$ is a disk.] A map $p: E \rightarrow B$ is a Serre microfibration if, for any homotopy $h: X \times[0,1] \rightarrow B$ with prescribed initial lift $\bar{h}_{0}: X \rightarrow E$, there exist a neighborhood $U$ of $X \times\{0\}$ in $X \times[0,1]$ and a map $\bar{h}: U \rightarrow E$ such that $p \bar{h}=h \mid U$ and $\bar{h}(x, 0)=\bar{h}_{0}(x)$ for all $x \in X$. In that case the map $\bar{h}$ is a microlift of $h$. [Again it is enough to check the micro-lifting property in all cases where $X$ is a disk.] 
Lemma 2.2. Let $p: E \rightarrow B$ be a Serre microfibration. If $p$ has weakly contractible fibers, then it is a Serre fibration.

Notes on the proof. This is essentially due to G. Segal [8, A.2]. The hypotheses here are slightly more general, though. There is a short inductive argument as follows. The induction step consists in showing that if $p: E \rightarrow B$ is a Serre microfibration with contractible fibers, then so is the projection $p^{I}: E^{I} \rightarrow B^{I}$. Here $I=[0,1]$, and the mapping spaces $E^{I}=\operatorname{map}(I, E)$ and $B^{I}=\operatorname{map}(I, B)$ come with the compactopen topology. The Serre microfibration property for $p^{I}$ is obvious, so it is enough to establish the weak contractibility of the fibers of $p^{I}$. Suppose therefore given a map $\gamma: I \rightarrow B$ and a map $f: \mathbb{S}^{n} \times I \rightarrow E$ which covers $\gamma$, so that $p f(z, t)=\gamma(t)$ for $z \in \mathbb{S}^{n}$ and $t \in I$. We must extend $f$ to a map $g: \mathbb{D}^{n+1} \times I \rightarrow E$ which covers $\gamma$. But that is easy: Use a sufficiently fine subdivision of $I$ into subintervals $\left[a_{r}, a_{r+1}\right]$ so that partial extensions

$$
g_{r}: \mathbb{D}^{n+1} \times\left[a_{r}, a_{r+1}\right] \rightarrow E
$$

of $f$ can be constructed, with $p g_{r}(z, t)=\gamma(t)$ for $z \in \mathbb{D}^{n+1}$ and $t \in\left[a_{r}, a_{r+1}\right]$. Then improve $g_{r}$ if necessary, on a small neighborhood of $\mathbb{D}^{n+1} \times\left\{a_{r}\right\}$ in $\mathbb{D}^{n+1} \times\left[a_{r}, a_{r+1}\right]$, to ensure that $g_{r}\left(z, a_{r}\right)=g_{r-1}\left(z, a_{r}\right)$ for $z \in \mathbb{D}^{n+1}$.

The induction beginning consists in showing that $p$ has the path lifting property. (That is, given a path $\gamma: I \rightarrow B$ and $a \in E$ with $p(a)=\gamma(0)$, there exists a path $\omega: I \rightarrow E$ with $p \omega=\gamma$ and $\omega(0)=a$.) But that is also easy.

Lemma 2.3. Let $\tau$ be the tautological functor from $\mathcal{O}(X)$ to spaces and let $K$ be a compact subset of hocolim $\tau$. Then there exist a finite full sub-poset $\mathcal{P} \subset \mathcal{O}(X)$ and a subfunctor $\kappa$ of $\tau \mid \mathcal{P}$ with compact values such that $K \subset$ hocolim $\kappa$.

Remarks. The fullness assumption means that $U, V \in \mathcal{P}$ and $U \subset V$ imply $U \leqslant V$ in $\mathcal{P}$. By a subfunctor $\kappa$ of $\tau \mid \mathcal{P}$ is meant a selection of subspaces $\kappa(U) \subset \tau(U)=U$, one for each $U \in \mathcal{P}$, such that $\kappa(V) \subset \kappa(U)$ if $V \leqslant U$ in $\mathcal{P}$.

The lemma is closely related to an observation for which I am indebted to Larry Taylor: The mapping cylinder $C$ of the inclusion of the open unit interval in the closed unit interval is not homeomorphic to a subset of $[0,1]^{2}$. This is easy to verify, although surprising. The two endpoints of the closed unit interval, viewed as elements of the mapping cylinder $C$, don't have countable neighborhood bases; hence $C$ is not even metrizable. Equally surprising, and more to the point, is the following. Let $K$ be a compact subset of $C$. Then there exists a compact subinterval $L$ of the open unit interval such that $K$ is contained in the mapping cylinder of the inclusion $L \rightarrow[0,1]$. For the proof, exhaust the open unit interval by an ascending sequence of compact subintervals $L_{i}$. Suppose if possible that for each $i$ there exists $x_{i} \in K$ which is not contained in the mapping cylinder of the inclusion $L_{i} \rightarrow[0,1]$. Then the $x_{i}$ form an infinite discrete closed subset of $K$, which contradicts the compactness of $K$.

Proof of lemma 2.3. The classifying space $B \mathcal{O}(X)$ is a simplicial complex. This has one $n$-simplex for each subset of $\mathcal{O}(X)$ of the form $\left\{U_{0}, U_{1}, \ldots, U_{n}\right\}$ where $U_{i-1}$ is a proper subset of $U_{i}$, for $i \in\{1,2, \ldots, n\}$. The image of $C$ under the projection 
hocolim $\tau \rightarrow B \mathcal{O}(X)$ is contained in a compact simplicial subcomplex $B \mathcal{O}(X)$, and without loss of generality we can assume that the subcomplex has the form $B \mathcal{P}$ for a finite full sub-poset $\mathcal{P}$ of $\mathcal{O}(X)$. For each simplex $S$ of $B \mathcal{P}$, let $e(S) \subset B \mathcal{P}$ be the "cell" determined by $S$, so that $e(S)$ is locally closed in $B \mathcal{P}$ and $B \mathcal{P}$ is the disjoint union (but not the coproduct in general) of the $e(S)$ for the simplices $S$ of $B \mathcal{P}$. Let $U(S)$ be the smallest of the open sets corresponding to the vertices of $S$. The inverse image of $e(S)$ for the projection

$$
\text { hocolim } \tau \longrightarrow B \mathcal{O}(X)
$$

is identified with $e(S) \times U(S)$. Its intersection with $K$ is contained in a subset of the form $e(S) \times L(S)$, where $L(S) \subset U(S)$ is compact. (This can be proved as in the remark just above.) Choose such an $L(S)$ for every simplex $S$ in $B \mathcal{P}$. For an element $U$ of $\mathcal{P}$ let

$$
\kappa(U):=\bigcup_{S \text { with } U(S) \subset U} L(S) .
$$

Then $\kappa(V) \subset \kappa(U)$ for $V, U \in \mathcal{P}$ with $V \subset U$, and each $\kappa(U)$ is compact.

Corollary 2.4. The projection $p_{\mathscr{F}}: X_{\mathscr{F}} \rightarrow X$ is a Serre microfibration.

Proof. Write $p=p_{\mathscr{F}}$. Let $q: X_{\mathscr{F}} \rightarrow B \mathcal{T}_{\mathscr{F}}$ be the standard projection (from the homotopy colimit to the classifying space of the indexing category). As we just discovered, the formula $y \mapsto(p(y), q(y))$ need not define an embedding of $X_{\mathscr{F}}$ in $X \times B \mathcal{T}_{\mathscr{F}}$, but it certainly defines an injective map and we can use that to label elements of $X_{\mathscr{F}}$. In particular, let $h: \mathbb{D}^{i} \times[0,1] \longrightarrow X$ be a homotopy with an "initial lift" $H_{0}: \mathbb{D}^{i} \longrightarrow X_{\mathscr{F}}$, so that $h(z, 0)=p H_{0}(z)$. We need to find $\varepsilon>0$ and a map $H: \mathbb{D}^{i} \times[0, \varepsilon] \longrightarrow X_{\mathscr{F}}$ such that $p H=h$ on $\mathbb{D}^{i} \times[0,1]$ and $H(z, 0)=H_{0}(z)$ for $z \in \mathbb{D}^{i}$. The plan is to define $H$ in such a way that

$$
(p H(z, t), q H(z, t))=\left(h(z, t), q H_{0}(z)\right),
$$

for $(z, t) \in \mathbb{D}^{i} \times[0, \varepsilon]$, which means that $q H: \mathbb{D}^{i} \times[0,1] \longrightarrow B \mathcal{T}_{\mathscr{F}}$ is a constant homotopy. By lemma 2.3, the plan is sound, giving a well defined and continuous map $\mathbb{D}^{i} \times[0, \varepsilon] \longrightarrow X_{\mathscr{F}}$ for sufficiently small $\varepsilon$.

Lemma 2.5. The projection $p_{\mathscr{F}}: X_{\mathscr{F}} \longrightarrow X$ has contractible fibers.

Proof. The fiber over $x \in X$ is identified with the homotopy colimit of the (contravariant, set-valued) functor

$$
(U, c) \mapsto \mathscr{F}^{(c)}(U)
$$

where $U$ runs through the open subsets of $X$ containing $x$, and $c$ runs through the objects of $\mathcal{C}$. By a well-known Fubini principle for homotopy colimits, it is homotopy equivalent to the double homotopy colimit

$$
\underset{c}{\operatorname{hocolim}} \underset{U \ni x}{\operatorname{hocolim}} \mathscr{F}^{(c)}(U) .
$$


In this expression the inside homotopy colimit is a homotopy colimit of sets (i.e., discrete spaces) taken over a directed poset, and therefore the canonical map

$$
\underset{U \ni x}{\operatorname{hocolim}} \mathscr{F}^{(c)}(U) \longrightarrow \operatorname{colim}_{U \ni x} \mathscr{F}^{(c)}(U)
$$

is a homotopy equivalence. Therefore

$$
\underset{c}{\operatorname{hocolim}} \underset{U \ni x}{\operatorname{hocolim}} \mathscr{F}^{(c)}(U) \simeq \underset{c}{\operatorname{hocolim}} \mathscr{F}_{x}^{(c)}
$$

where $\mathscr{F}_{x}^{(c)}$ is the stalk of $\mathscr{F}^{(c)}$ at $x$. But the stalk functor $\mathscr{F}_{x}$ is representable by assumption. The homotopy colimit of a representable functor is contractible.

Proof of proposition 2.1. Apply lemma 2.2 and note that a Serre fibration with weakly contractible fibers is a weak homotopy equivalence.

\section{Classification of sheaves up to concordance}

Lemma 3.1. Let $\mathscr{F}_{0}$ and $\mathscr{F}_{1}$ be two sheaves of $\mathcal{C}$-sets on $X$, both with representable stalks. Let $g: \mathscr{F}_{0} \rightarrow \mathscr{F}_{1}$ be a binatural transformation. Then $\mathscr{F}_{0}$ and $\mathscr{F}_{1}$ are concordant.

Proof. Let $e_{0}: X \rightarrow X \times I$ be given by $e_{0}(x)=(x, 0)$ and let $p: X \times I \rightarrow X$ be the projection. For an object $c$ in $\mathcal{C}$ and an open subset $U$ of $X \times[0,1]$, let $U_{0}=e_{0}^{-1}(U)$ and let $\mathscr{G}^{(c)}(U)$ be the set of pairs $(s, t) \in \mathscr{F}_{0}\left(U_{0}\right) \times p^{*} \mathscr{F}_{1}(U)$ such that $g s=e_{0}^{*} t$. Now $\mathscr{G}$ is a sheaf of $\mathcal{C}$-sets on $X \times I$ with representable stalks. Its restrictions to $X \times\{0\}$ and $X \times\{1\}$ are identified with $\mathscr{F}_{0}$ and $\mathscr{F}_{1}$, respectively.

Corollary 3.2 (to proposition 2.1 and lemma 3.1). Let $\mathscr{F}$ be a sheaf of $\mathcal{C}$-sets on $X$ with representable stalks. Suppose that $X$ is a $C W$-space. Then

$$
\pi_{\mathscr{F}} p_{\mathscr{F}}^{-1}: X \rightarrow B \mathcal{C}
$$

is a classifying map for $\mathscr{F}$. That is, $\left(\pi_{\mathscr{F}} p_{\mathscr{F}}^{-1}\right) * \mathscr{E}$ is concordant to $\mathscr{F}$, with $\mathscr{E}$ as in definition 1.1.

Proof. Abbreviate $p=p_{\mathscr{F}}, \pi=\pi_{\mathscr{F}}$. It is enough to show that the sheaves $\pi^{*} \mathscr{E}$ and $p^{*} \mathscr{E}$ on $X_{\mathscr{F}}$ are concordant. By lemma 3.1, it is then also enough to make a map from $\pi^{*} \mathscr{E}$ to $p^{*} \mathscr{F}$. That is what we will do, using the "étale" point of view. Therefore let $z \in X_{\mathscr{F}}$. We need to compare the stalk of $\mathscr{F}$ at $p(z) \in X$ with the stalk of $\mathscr{E}$ at $\pi(z) \in B \mathcal{C}$. The point $z$ maps to some cell in $B \mathcal{T}_{\mathscr{F}}$ which corresponds to a nondegenerate diagram

$$
\left(U_{0}, c_{0}\right) \leftarrow\left(U_{1}, c_{1}\right) \leftarrow \cdots \leftarrow\left(U_{k-1}, c_{k-1}\right) \leftarrow\left(U_{k}, c_{k}\right)
$$

in $\mathcal{O}(X) \times \mathcal{C}$, with $p(z) \in U_{k}$, and an element $s_{0} \in \mathscr{F}\left(c_{0}\right)\left(U_{0}\right)$. The stalk of $\mathscr{E}$ at $\pi(z)$ is then represented by the object $c_{0}$. The germ of $s_{0}$ near $p(z)$ amounts to a morphism from $c_{0}$ to the object which represents the stalk of $\mathscr{F}$ at $p(z)$; equivalently, by the Yoneda lemma, $s_{0}$ determines a $\mathcal{C}$-map from the stalk of $\mathscr{E}$ at $\pi(z)$ to the stalk of $\mathscr{F}$ at $p(z)$. Letting $z$ vary now, and selecting an object $c$ in $\mathcal{C}$, we obtain a map over $X_{\mathscr{F}}$ from $\operatorname{Spé}\left(\pi^{*} \mathscr{E}(c)\right)$ to $\operatorname{Spé}\left(p^{*} \mathscr{F}^{(c)}\right)$. This is continuous (verification left 
to the reader) and natural in $c$, and therefore amounts to a map between sheaves of $\mathcal{C}$-sets on $X_{\mathscr{F}}$, from $\pi^{*} \mathscr{E}$ to $p^{*} \mathscr{F}$.

Proof of theorem 0.1. Suppose that $X$ is a CW-space. Let $g: X \rightarrow B \mathcal{C}$ be any map and put $\mathscr{F}=g^{*} \mathscr{E}$. We have to show that $g$ is homotopic to $\pi p^{-1}$, where $\pi=\pi_{\mathscr{F}}$ and $p=p_{\mathscr{F}}$. We note that $X_{\mathscr{F}}$ also has the homotopy type of a CW-space since it is a homotopy colimit of open subsets of $X$ (all of which have the homotopy type of CW-spaces). Hence $p$ is a homotopy equivalence. Therefore, showing $g \simeq \pi p^{-1}$ amounts to showing that $g p \simeq \pi$.

Now recall that $X_{\mathscr{F}}$ was constructed as the homotopy colimit of a functor $\tau \varphi$ from a certain category $\mathcal{T}_{\mathscr{F}}$ with objects $(U, c, s)$ to the category of spaces. The maps $g p: X_{\mathscr{F}} \rightarrow B \mathcal{C}$ and $\pi: X_{\mathscr{F}} \rightarrow B \mathcal{C}$ both have a factorization of the following kind:

$$
\underset{(U, c, s)}{\operatorname{hocolim}} U \stackrel{v}{\longrightarrow} \underset{c}{\operatorname{hocolim}} \operatorname{Spé}(\mathscr{E}(c)) \stackrel{w}{\longrightarrow} B \mathcal{C} .
$$

Here $v$ is (in both cases) induced by a natural transformation from the functor $(U, c, s) \mapsto U$ to the functor $(U, c, s) \mapsto \operatorname{Spé}\left(\mathscr{E}^{(c)}\right)$, given by the maps

$$
U \longrightarrow \operatorname{Spé}\left(\mathscr{E}^{(c)}\right) \quad ; \quad x \mapsto(x, s) .
$$

In the case of $g p$, the second map $w=w_{0}$ in the factorization is determined by the projections $\operatorname{Spé}(\mathscr{E}(c)) \rightarrow B \mathcal{C}$. In the case of $\pi$, the map $w=w_{1}$ is the composition of the projection to $B \mathcal{T}_{\mathscr{F}}$ and the forgetful map $B \mathcal{T}_{\mathscr{F}} \rightarrow B \mathcal{C}$.

Consequently it is now sufficient to show that $w_{0}$ and $w_{1}$ are weakly homotopic, which is to say, $w_{0} f \simeq w_{1} f$ for any map $f$ from a $C W$-space to the common source of $w_{0}$ and $w_{1}$. It is enough to check this for a particular $f$ which is a weak equivalence. A good choice of such an $f$ is the map

$$
\text { hocolim } B(c \downarrow \mathcal{C}) \longrightarrow \operatorname{hocolim} \operatorname{Spé}\left(\mathscr{E}^{(c)}\right)
$$

induced by the natural maps $\lambda_{c}: B(c \downarrow \mathcal{C}) \rightarrow \operatorname{Spé}\left(\mathscr{E}^{(c)}\right)$. By proposition 1.3, this $f$ is indeed a weak homotopy equivalence. The maps $w_{0} f$ and $w_{1} f$ are easily seen to be homotopic. Indeed, each $(c \downarrow \mathcal{C})$ has two obvious functors two $\mathcal{C}$, one given by $(c \rightarrow d) \mapsto d$ and the other by $(c \rightarrow d) \mapsto c$. These are related by a natural transformation, which determines a homotopy $h^{(c)}$ between the induced maps $B(c \downarrow \mathcal{C})$ to $B \mathcal{C}$. Integrating the homotopies $h^{(c)}$ one obtains a homotopy $w_{0} f \simeq w_{1} f$.

\section{References}

[1] A. Dold, Lectures on algebraic topology, Grundlehren, Springer, 1972.

[2] R. Hartshorne, Algebraic geometry, Graduate Texts in Mathematics, Springer, 1977.

[3] J.P.May, Simplicial objects in algebraic topology, Chicago Lectures in Math., Univ. of Chicago Press, 1967.

[4] S. Mac Lane and I. Moerdijk, Sheaves in geometry and logic, Springer, 1992. 
[5] I. Madsen and M. Weiss, The stable moduli space of Riemann surfaces: Mumford's conjecture, preprint, arXiv:math.AT/0212321, v3, 2002-2004.

[6] I. Madsen and M. Weiss, The stable mapping class group and stable homotopy theory, Proceedings of 2004 European Cong. of Maths. in Stockholm, European Math. Soc., 2005.

[7] I. Moerdijk, Classifying spaces and classifying topoi, Lecture Notes in Math., vol. 1616, Springer, 1995.

[8] G. B. Segal, Classifying spaces related to foliations, Topology 17 (1978), 367382 .

This article is available at http://intlpress.com/HHA/v7/n1/a10/

Michael Weiss m.weiss@maths.abdn.ac.uk

Dept. of Mathematics,

University of Aberdeen,

AB24 3UE

United Kingdom 